\title{
Les antioncogènes
}

\begin{abstract}
Plusieurs types de cancers héréditaires sont associés spécifiquement à une délétion de certaines régions chromosomiques. Ces régions portent des gènes, différents des oncogènes, dont la fonction serait d'assurer la régulation de la différenciation et le contrôle de la prolifération cellulaire, peut-être en réprimant certains oncogènes, ce qui explique le nom proposé d'antioncogènes.
\end{abstract}

\section{Claudine Junien}

Maître de conférence des universités-praticien hospitalier. Unité Inserm $U 73$ et laboratoire de biochimie-génétique, hôpital Ambroise Paré.

\section{ADRESSE}

C. Junien: Unité Inserm U 73 et laboratoire de biochimie-génétique, hôpital Ambroise-Paré, Château de Longchamp, Bois de Boulogne, 75016 Paris. 'existence de modifications somatiques du matériel génétique dans le développement des cancers a été suggérée dès le début de ce siècle [I]. Ce n'est cependant qu'avec le développement spectaculaire des techniques de biologie moléculaire que certaines recherches ont pu aboutir, en particulier à la mise en évidence des gènes cellulaires baptisés "oncogènes ". Il a alors été possible de reconnaitre l'existence d'étapes successives dans le développement des cancers. Ces étapes différentes définissent des voies distinctes de cancérisation selon l'agent transformant (carcinogène chimique ou virus) ou l'oncogène impliqué ou bien encore le type cellulaire concerné.

L'analyse au niveau moléculaire des oncogènes activés présents dans les tumeurs a montré l'existence de différents types de mutation. Plusieurs mécanismes peuvent être responsables de la transition protooncogène cellulaire $\rightarrow$ oncogène activé: insertion d'un rétrovirus tumorigène, amplification génique, mutation ponctuelle, translocation à proximité d'une région transcriptionnellement active.

Si la signification fonctionnelle de ces altérations est loin d'être entièrement élucidée, cela provient pour une grande part de l'ignorance du rôle physiologique exact des pro- téines codées par ces gènes. Il ne fait aucun doute pourtant que l'orchestration harmonieuse de la vie de la cellule, par un contrôle de sa croissance et de sa division, et ce au cours des différentes étapes de son existence, repose sur des interactions subtiles auxquelles ces protéines (et bien d'autres également) participent et dont on commence à déchiffrer peu à peu la nature exacte.

Une remarque importante concernant le mode d'action de ces activations d'oncogènes s'impose: les oncogènes mutés se comportent à première vue au niveau cellulaire comme des allèles dominants. Un exemple bien connu est celui des mutations ponctuelles des oncogènes de la famille ras en position I2 ou 6r qui confêrent à elles seules le caractère transformant, et cela malgré la persistance de l'allèle normal qui ne suffit donc pas à maintenir le phénotype normal.

Dans la leucémie myéloïde chronique (LMC) et dans le lymphome de Burkitt*, l'expression des oncogènes situés au niveau des points de cassure sur les chromosomes $9(c$-abl) et $8(c-\mathrm{myc})$ est soit qualitativement, soit quantitativement modifiée. Il n'existe toutefois, à l'heure actuelle, aucun argument permettant de réfuter la mise en cause d'un mécanisme de type exclusion allélique. Il était donc tentant de rechercher pour 
chaque type de cancer l'oncogène "coupable " qui serait précisément situé au niveau de l'anomalie chromosomique spécifique. Or, un certain nombre de tumeurs dont font partie les tumeurs héréditaires et, plus particulièrement, les tumeurs embryonnaires, semblaient échapper à la logique de ce modèle.

\section{Prédisposition héréditaire}

Pour chaque type de cancer, on connaît des formes héréditaires et des formes sporadiques. Les premières se transmettent de façon dominante sous la forme d'une simple prédisposition: dans une famille donnée, les individus porteurs obligatoires de la mutation ne développent pas nécessairement une tumeur mais la probabilité est considérablement accrue. De plus, cette prédisposition peut être restreinte, dans certains cas, à un seul type de tumeur, ou bien, au contraire, concerner un ou plusieurs autres types de tissu, selon les individus.

Dans les cancers héréditaires, l'implication de gènes agissant selon un schéma différent de celui des oncogènes repose principalement sur deux types d'observations qui sont apparemment en conflit avec le modèle d'action des oncogènes. Alors qu'un oncogène activé peut produire un cancer à l'état hétérozygote, dans les cancers embryonnaires associés à une délétion chromosomique, comme le rétinoblastome $(\mathrm{Rb})$ ou le néphroblastome (tumeur de Wilms :WT), on a pu constater la perte ou l'altération de deux copies des gènes impliqués. Ceci suppose donc un mode d'action récessif. Par ailleurs, de nombreuses expériences de fusion cellulaire réalisant des hybrides somatiques entre des cellules transformées et des cellules normales ont montré que ces dernières possédaient des séquences capables de faire régresser le phénotype transformé [2].

Comme cela a été suggéré par Commings, en I973 [3], des loci récessifs de ce type, dont la fonction est apparemment perdue au cours du développement tumoral, pourraient correspondre: a) à des gènes de structure dont les produits agissent directement pour empêcher la croissance autonome de cellules normales ou bien : b) à des gènes régulateurs dont la fonction serait de supprimer celle d'autres gènes, comme les oncogènes, codant pour des facteurs transformants. Un tel mode de régulation négative opérerait au niveau transcriptionnel [4]. Mais il n'est pas impossible que d'autres gènes agissent aussi au niveau post-traductionnel en supprimant l'effet du produit d'un oncogène [5]. La spécificité du mode d'action de ces gènes ressort également d'expériences d'hybridation somatique [6].

\section{Les tumeurs embryonnaires}

Le rétinoblastome appartient, comme le néphroblastome, le neuroblastome et d'autres tumeurs de l'enfant [7], à la catégorie des tumeurs embryonnaires. Ces tumeurs apparaissent chez l'enfant jeune, sont souvent associées à des anomalies spécifiques comme l'hémihypertrophie et semblent souvent consécutives à une hyperplasie cellulaire qui exagère peut-être la potentialité maligne des cellules. Ces tumeurs embryonnaires se développent aux dépens de cellules très jeunes analogues à celles des stades précoces de l'embryogénèse.

Ce sont des tumeurs rares: I cas sur 20000 naissances pour le rétinoblastome (Rb), et I sur I o ooo pour la tumeur de Wilms (WT), ou néphroblastome. On connaît des formes sporadiques unilatérales (les plus fréquentes), des formes familiales souvent bilatérales (au moins ro $\%$ mais peut-être plus) et un faible pourcentage de cas associés à une délétion chromosomique plus ou moins étendue selon les individus mais ayant en commun une petite partie de la région I 3 qI 4 pour le Rb et I I pI 3 pour la WT. L'examen minutieux des points de cassure observés chez les différents individus a montré qu'il ne pouvait pas y avoir de point de cassure commun, excluant donc l'intervention d'un oncogène au niveau des points de cassure.

Il a été montré que des séquences situées en I 3 q I 4 jouaient également un rôle déterminant dans le déve- loppement des tumeurs sporadiques puisque des anomalies chromosomiques impliquant cette région du I 3 étaient retrouvées a u niveau tumoral chez des sujets présentant un caryotype constitutionnel normal. De même, la participation de ces séquences à l'apparition des formes familiales a été démontrée par la mise en évidence d'une liaison génétique avec le marqueur estérase $D$ (ESD) localisé lui-même en I3 q I4. Il a été démontré, de la même façon, que la région i I p I 3 est également impliquée dans les formes sporadiques de tumeur de Wilms. Selon Knudson [8], le développement de la tumeur nécessiterait deux mutations successives pour une même cellule. Ces deux mutations peuvent être somatiques, la tumeur est alors sporadique et unilatérale. Par contre, si la première mutation survient dans une cellule germinale, il suffira d'une deuxième mutation pour déclencher l'apparition de la tumeur plus facilement multiple (multifocale et/ou bilatérale).

\section{Le modèle rétinoblastome}

La perte de l'hétérozygotie. Dès I983, Benedict et coll. et Godbout et coll. montraient chez un de leurs malades atteint de $\mathrm{R} b$, la perte, dans les cellules tumorales, du deuxième allèle correspondant au gène "suppresseur" $R b^{\mathbf{s}}[9$, ro]. Ce sujet présentait, au niveau constitutionnel, une délétion submicroscopique de la bande I 3 qI 4 révélée par une diminution de $50 \%$ de l'activité $\mathrm{ESD}$, marqueur de cette région. Au niveau tumoral, l'absence d'activité ESD indiquait la perte du chromosome normal, l'unique chromosome I3 présent dans les cellules tumorales devant donc correspondre au chromosome I 3 délété.

Cette hypothèse devait être confirmée par les travaux de Cavenee $e t$ coll. [I I] qui, grâce à des marqueurs polymorphes de l'ADN situés dans le bras long du chromosome $\mathrm{r} 3$, et pour lesquels les sujets étudiés étaient hétérozygotes de façon constitutionnelle, devaient démontrer la nature des événements mitotiques conduisant à la perte des deux allèles ou bien à la réalisation d'une homozygotie pour le gène muté $(f-$ 
gure I). Plusieurs types d'événements ont été démontrés: non-disjonction, suivie d'une reduplication du chromosome restant porteur de l'allèle muté et recombinaison mitotique, pour environ la moitié des sujets examinés.

D'autres types d'événements [12] aboutiraient également au même résultat mais ne peuvent pas encore être mis en évidence en l'absence de sondes d'ADN suffisamment proches du locus $\mathrm{Rb}^{s}$ : mutation ponctuelle, conversion génique, microdélétion. Bien qu'il ne soit pas possible de repousser l'éventualité de l'existence d'autres loci pour le $R b$, il est vraisemblable que ces types d'événements se produisent et rendent compte du fait que, sur l'ensemble des tumeurs examinées et comparées au génotype correspondant de l'individu, la moitié seulement d'entre elles montraient une perte de l'hétérozygotie pour les marqueurs du I 3 étudiés.

Ceci est parfaitement en accord avec la théorie proposée par Knudson: deux événements sont nécessaires à l'apparition d'une tumeur, le premier d'entre eux pouvant être somatique ou bien constitutionnel (cas familiaux ou avec délétion), le deuxième étant somatique.

\section{REFERENCES}

1. Boveri T. Zur Frage der Entstehung Malinger Tumoren. Jean, Germany: Fischer I9I4.

2. Croce CM. Cancer genes in cell hybrids. Biochim et Biophys Acta 1980; 605: 411-30.

3. Commings DE. A general theory of carcinogenesis. Proc Natl Acad Sci USA I 973; 70: 3324-8.

4. Dyson PJ, Quade K, Wyke JA., Expression of the ASV src gene in hybrids between normal and virally transformed cells: 3 Specific suppression occurs in some hybrids but not others. Cell 1982 ; 36: 49 I-8.

5. Craig RW, Sager R. Suppression of tumorigenicity in hybrids of normal and oncogenetransformed CHEF cells. Proc Natl Acad Sci $U S A$ 1985; 82: 2062-6.

6. Noda M, Selinger Z, Scolnick EM, Bassin RH. Flat revertants isolated from Kirsten sarcoma virus-transformed cells are resistant to the action of specific oncogenes. Proc Natl Acad Sci USA I983; 80: 5602-6.

7. Schweisguth O. Les tumeurs solides de l'enfant. Paris: Flammarion, 1979: 317 pages.

8. Knudson AG. Mutation and cancer: statistical study of retinoblastoma. Proc Natl Acad Sci $U S A$ I97 1; 68: 820-3.

9. Benedict WF, Murphree AL, Banerjee A, Spina CA, Sparkes MC, Sparkes RS. Patient with I3 chromosome deletion: evidence that the retinoblastoma gene is a recessive cancer gene. Science I983; 2 19: $973-4$

Io. Godbout R, Dryja TP, Squire J, Gallie BL, Phillips RA. Somatic inactivation of genes on chromosome 13 is a common event in retinoblastoma. Nature 1983; 304: 45I-3.

I I. Cavenee WK, Dryja TP, Phillips RA, et al. Expression of recessive alleles by chromosomal mechanisms in retinoblastoma. Nature $1983 ; 305$ : 779-84.

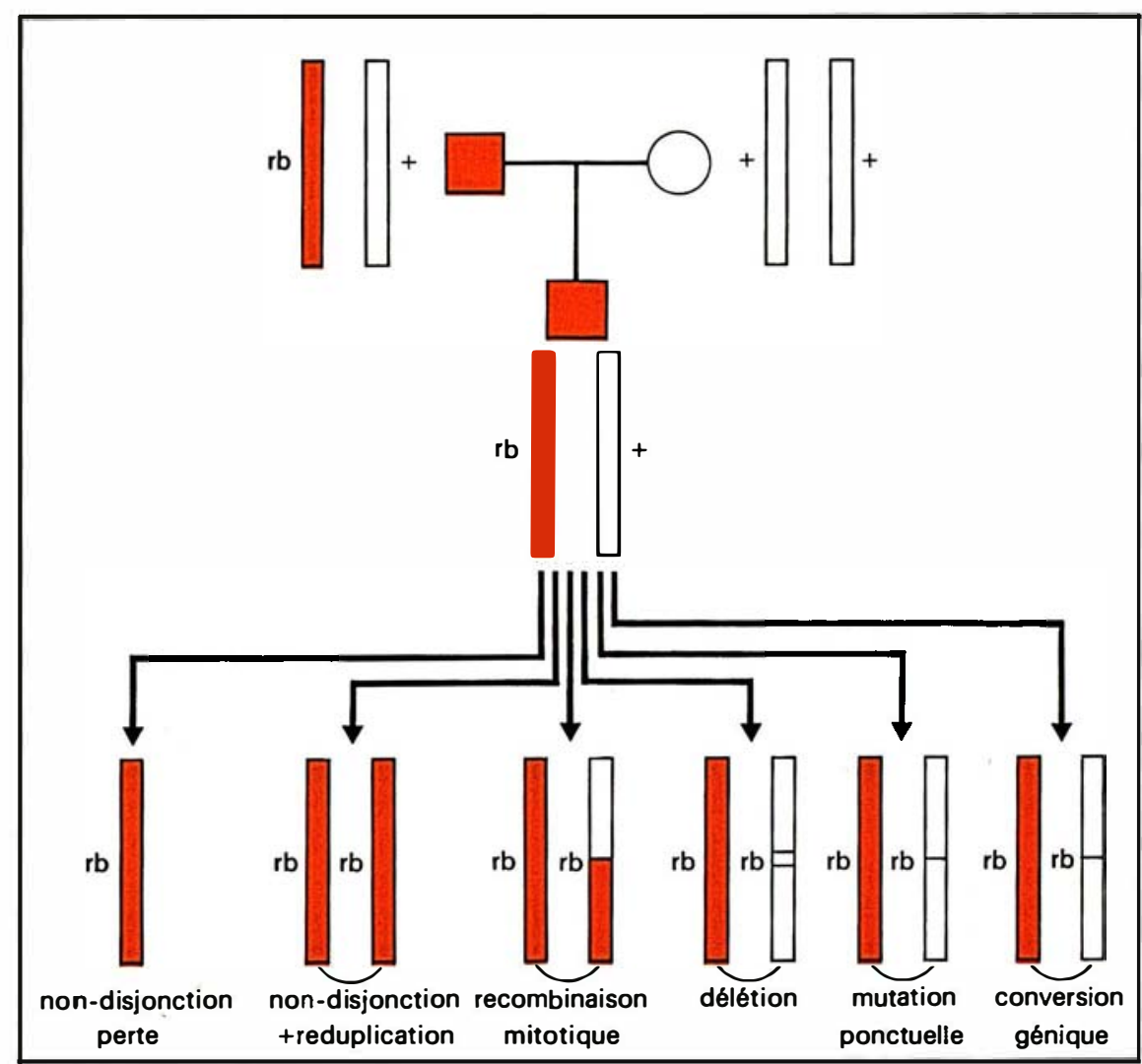

Figure 1. Mécanismes mitotiques réalisant, au niveau cellulaire (tumoral), 1) l'hémizygotie au locus $R b^{s}$, dans le cas de la perte d'un chromosome 13 entier ou de la région rb, soit par non-disjonction, soit par délétion; 2) l'homozygotie dans les cinq autres types d'événements mitotiques. Cette perte de l'hétérozygotie, qui aboutit à une inactivation somatique de l'allèle normal (+) chez un sujet ayant hérité de la mutation rb, peut être mise en évidence grâce à des marqueurs de l'ADN qui permettent de différencier le chromosome d'origine paternelle portant une mutation au locus rb (chromosome rouge) du chromosome d'origine maternelle (chromosome blanc) portant l'allèle normal (+) qui sera inactivé dans les cellules de la rétine. 
Spécificité chromosomique. Il a de plus, été démontré, en utilisant des marqueurs correspondant à d'autres chromosomes, que seul le chromosome 13 était touché.

Spécificité tissulaire. Plusieurs autres types de tumeurs ont été étudiées avec les marqueurs du I 3 et d'autres chromosomes: sarcome d'Ewing*, néphroblastome, rhabdomyosarcome*, hépatoblastome, lymphome de Burkitt. Ces tumeurs ne montrent pas de perte de l'hétérozygotie pour le chromosome i 3 .

Mécanisme commun au rétinoblastome et à l'ostéosarcome. Pourtant un autre type de tumeur, l'ostéosarcome, dépend du même mécanisme (perte de l'hétérozygotie pour le chromosome 13). Or, cette tumeur osseuse est liée au rétinoblastome puisqu'elle apparaît souvent comme seconde tumeur chez les sujets atteints de rétinoblastome. On connaît également des cas familiaux d'ostéosarcomes dans des familles avec rétinoblastome. Cette découverte démontre donc l'existence d'un mécanisme commun à ces deux types de tumeur [13].

\section{Le syndrome WAGR}

L'histoire de la tumeur de Wilms montre de grandes similitudes avec celle du rétinoblastome. On distingue également différentes formes étiologiques; cependant la proportion de cas familiaux reconnus ou de cas avec délétion semble plus réduite pour le néphroblastome. Très tôt, les pédiatres suivant les enfants atteints d'un néphroblastome avaient remarqué une fréquence, beaucoup plus élevée que dans la population normale, d'enfants aniridiques*. L'aniridie sporadique qui survient une fois sur 50000 naissances est observée dans I sur 75 néphroblastomes. L'examen caryotypique de ces sujets montre une délétion chromosomique, cette fois de la région proximale de la bande i i p i3. La délétion s'accompagne d'anomalies génito-urinaires avec ambiguité sexuelle, d'hémihypertrophie et d'arriération mentale. Le sigle WAGR regroupe les anomalies caractéristiques de ce syndrome: $\mathrm{W}$ pour prédisposition à la tumeur de Wilms, A pour aniridie, $G$ pour anomalies génito-urinaires, $R$ pour arriération mentale. L'étude cytogénétique d'autres enf ants présentant une aniridie et ces anomalies associées mais sans tumeur, a permis de connaitre la proportion d'enfants chez lesquels cette délétion entraîne l'apparition d'un néphroblastome: plus d'un tiers des cas. Cette proportion semble beaucoup plus importante pour le rétinoblastome.

La perte de l'hétérozygotie. Plusieurs groupes ont analysé les polymorphismes de l'ADN détectés par différents marqueurs localisés sur le bras court du chromosome in [i4I 8]. La comparaison du génotype des individus étudiés et des allèles correspondants présents dans la tumeur a révélé les mêmes types d'événements mitotiques, c'est-àdire non-disjonction suivie d'une reduplication du chromosome restant, ou bien recombinaison mitotique, dans environ la moitié des cas. Spécificité chromosomique. L'étude de marqueurs correspondant à d'autres chromosomes a confirmé la spécificité de l'anomalie touchant le chromosome i I puisqu'aucun événement mitotique de ce type ne semble avoir touché d'autres chromosomes.

Spécificité tissulaire. D'autres tumeurs ont été analysées à l'aide de marqueurs du il et d'autres chromosomes (sarcome d'Ewing, $\mathrm{Rb}$, neuroblastome, ostéosarcome): aucune d'entre elles ne montre une perte de l'hétérozygotie au niveau du chromosome i I. Pourtant, comme nous allons le voir dans la suite du texte, il semble qu'à l'instar du rétinoblastome et de l'ostéosarcome, il existe un mécanisme commun à la WT et à d'autres tumeurs.

\section{Syndromes associés au néphroblastome}

Les travaux de ces différentes équipes montrent bien qu'une grande partie (et peut-être la totalité) des néphroblastomes se caractérisent par la perte de l'hétérozygotie au niveau du chromosome II, qu'il s'agisse de formes sporadiques ou de formes héréditaires associées à la délétion chromosomique I I pi3. Cet événement constitue vraisemblablement l'une des étapes indispensables caractéristique de la transformation maligne.

Le néphroblastome se caractérise par la présence de trois types cellulaires des stades précoces du développement embryonnaire rénal reproduisant l'aspect du métanephros: cellules très peu différenciées mais probablement déjà "induites", de type blastème; cellules de type conjonctif et enfin cellules de type épithélial qui participent toutes, en coopération avec les cellules du bourgeon urétéral, à l'élaboration du néphron. La présence de trois types de cellules qui se retrouvent seules ou en association dans la zone tumorale contraste avec l'aspect mature totalement différencié de la zone adjacente du rein normal. Malgré l'aspect différencié du rein humain après la naissance, on ne peut exclure, comme chez le rat nouveau-né, la persistance d'un très petit nombre de cellules blastémateuses. L'inactivation totale du gène suppresseur dans ce type de cellule peut en favoriser la croissance et aboutir à une déviation du cycle normal de différenciation. Une autre explication, celle d'une dé-différenciation, plus ou moins aćcentuée selon les cas, permettrait de rendre compte de la présence simultanée ou non de différents types cellulaires correspondant à des stades plus ou moins avancés de différenciation. On sait que l'induction des cellules du blastème ne peut se faire sans un contact avec des cellules d'origine différente, celles du bourgeon urétéral. On ignore tout cependant quant aux bases moléculaires de ces "contacts" et s'il en existe d'autres. Les cellules peu différenciées retrouvées dans la tumeur se trouvent donc peut-être totalement isolées, séparées des autres cellules qui sont leurs partenaires normaux au cours de l'embryogénèse et, privées des signaux qui leur sont indispensables, se transf orment en cellules malignes.

Mécanisme commun au néphroblastome, à l'hépatoblastome et au rhabdomyosarcome.

La perte de l'hétérozygotie au niveau du chromosome i I est également retrouvée dans d'autres types de tumeurs rares de l'enfant, l'hépatoblastome et le rhabdomyosarcome. Ces tissus, foie et muscle strié d'une part, rein d'autre part, qui ne 


\section{REFERENCES}

12. Turner DR, Morley AA, Haliandros M, Kutlaca $R$, Sanderson BJ. In vivo somatic mutations in human lymphocytes frequently result from major gene alterations. Nature 1985; 315 : 343-5.

13. Hansen MF, Koufos A, Gallie BL, et al. Osteosarcoma and retinoblastoma: a shared chromosomal mechanism revealing recessive predisposition. Proc Natl Acad Sci USA 1985; 82: 1-5.

14. Koufos A, Hansen MF, Lampkin BC, et al. Loss of alleles at loci on human chromosome iI during genesis of Wilms' tumour. Nature 1984; 309: 170-2.

15. Orkin SH, Goldman DS, Sallan SE. Development of homozygosity for chromosome in $p$ markers in Wilms'tumour. Nature 1984; 309: 172-4.

16. Reeve AE, Housiaux PHJ, Gardner RJM, Chewings WE, Grindley RM, Millow L J. Loss of a harvey ras allele in sporadic Wilms' tumor. Nature 1984; 309: 174-6.

17. Fearon ER, Vogelstein B, Feinberg AP. Somatic deletion and duplication of genes on chromosome 11 in Wilms' tumour. Nature 1984; 309: 176-7.

18. Huerre C, Despoisse S, Gilgenkrantz S, Lenoir GM, Junien C. c-Ha-ras I is not deleted in aniridia-Wilms' tumour association. Nature 1983; 305: 638-4I.

19. Koufos A, Hansen MF, Copeland NG, Jenkins NA, Lampkin BC, Cavenee WK. Loss of heterozygosity in three embryonal tumours suggests a common pathogenetic mechanism. Nature 1985; 316: 330-4.

20. Turleau C, de Grouchy J. BeckwithWiedemann syndrome. Clinical comparison between patients with and without II p 15 trisomy. Ann Genet 1985; 28: 93-6.

21. Turleau C, de Grouchy J, Tournade MF, Gagnadoux MF, Junien C. Del I p/aniridia complex. Report of three patients and review of 37 observations from the literature. Clin Genet 1984; 26: 356-62.

22. Eddy AA, Mauer AM. Pseudohermaphroditism, glomerulopathy and Wilms tumor (Drash syndrome). Frequency in end-stage renal failure. f Pediatrics 1985; 106: 584-7.
Tableau I

SYNDROMES AVEC PRÉDISPOSITION

A LA TUMEUR DE WILMS (WT)

\begin{tabular}{|c|c|c|c|}
\hline . & WAGR & $\begin{array}{l}\text { Beckwith- } \\
\text { Wiedemann } \\
\text { (BW) }\end{array}$ & Drash \\
\hline $\begin{array}{l}\text { ANOMALIE } \\
\text { TIONNELLE }\end{array}$ & $11 \stackrel{\mathrm{p} 13}{\mathrm{del}}$ & $\begin{array}{c}\text { dupl } \\
11 \text { pter } \rightarrow 11 \text { p } 15.4\end{array}$ & $?$ \\
\hline MARQUEURS & $\begin{array}{l}\text { catalase, } \\
\text { FSH }\end{array}$ & $\begin{array}{c}\text { INS, RAS, IGF } 2 \text {, } \\
\text { HBB, TH }\end{array}$ & $?$ \\
\hline $\begin{array}{l}\text { SYNDROME } \\
\text { analogies } \\
\text { arriération mentale } \\
\text { hémihypertrophie } \\
\text { ambiguité sexuelle } \\
\text { - spécificités } \\
\text { aniridie } \\
\text { macroglossie } \\
\text { omphalocèle (voir } \\
\text { glossaire) } \\
\text { grande taille } \\
\text { glomérulopathie }\end{array}$ & $\begin{array}{l}+ \\
+ \\
+ \\
+\end{array}$ & + & + \\
\hline $\begin{array}{l}\text { TUMEUR } \\
2^{\mathrm{e}} \text { tumeur ou tumeur } \\
\text { plus rare }\end{array}$ & $\begin{array}{c}\text { WT } \\
\text { gonado- } \\
\text { blastome }\end{array}$ & $\begin{array}{c}\text { WT } \\
\text { hépatoblastome } \\
\text { rhabdomyosarcome } \\
\text { carcinome } \\
\text { surrénalien } \\
\text { gonadoblastome }\end{array}$ & $\begin{array}{l}\text { WT } \\
\text { gonado- } \\
\text { blastome }\end{array}$ \\
\hline
\end{tabular}

Syndromes prédisposant à l'apparition d'un néphroblastome. L'existence d'un petit nombre de cas présentant ces syndromes en association avec une anomalie chromosomique constitutionnelle a permis de connaître les régions impliquées dans le WAGR et le BW. Différents marqueurs ont été localisés dans ces régions et ont été utilisés pour étudier les différentes formés étiologiques (FSH =folliculo-stimulating-hormone, INS =insuline, $R A S=c$-Harvey-ras, $H B B=\beta$ globine, $T H=$ tyrosine hydroxylase). Ces syndromes, outre la prédisposition à la WT, montrent quelques ressemblances dont certaines comme l'arriération mentale sont vraisemblablement la conséquence du déséquilibre chromosomique. Certains signes cliniques comme l'aniridie peuvent être considérés comme indépendants de la prédisposition à la WT puisqu'elle résulte de la délétion simultanée d'un locus proche mais indépendant du gène WTs. Bien qu'un mécanisme commun au néphroblastome, au rhabdomyosarcome et à l'hépatoblastome ait été démontré [19], le ou les gènes impliqués dans l'apparition des tumeurs spécifiques de ces syndromes ont vraisemblablement la même conséquence phénotypique mais pourraient agir à un niveau différent, puisque les gènes impliqués dans l'hépatoblastome ou le rhabdomyosarcome sont épargnés dans les syndromes WAGR et de Drash. del=délétion; dup/=duplication. 
présentent pas d'origine embryonnaire commune, partagent vraisemblablement une étape commune au cours de leur différenciation. En effet, l'examen histologique de ces tumeurs a montré, dans certains cas, la juxtaposition dans des hépatoblastomes, des rhabdomyosarcomes ou des néphroblastomes, des structures cellulaires correspondant à un aspect d'hépatocyte, de muscle strié, ou de néphron [19]. De plus, ces différents types de tumeur peuvent apparaître indifferemment chez les enfants présentant un syndrome de Beckwith-Wiedemann (BW) avec, toutefois, une plus forte prédisposition pour le néphroblastome puisque $55 \%$ des enfants atteints de $\mathrm{BW}$ et présentant une tumeur avaient un néphroblastome [20]. Les travaux récents de Koufos et coll. [19] confirment cette hypothèse de l'étape commune au processus de différenciation de ces trois tissus différents, puisque la perte de l'hétérozygotie au niveau du chromosome I I se retrouve dans des hépatoblastomes et des rhabdomyosarcomes.

Il n'en demeure pas moins que l'on s'explique mal pourquoi la délétion I $\mathrm{pI} 3$ avec le syndrome WAGR d'une part [2I], et le syndrome de Drash d'autre part [22], syndrome caractérisé par une ambiguïté sexuelle et une glomérulopathie sévère, ne prédisposent qu'au néphroblastome ou, dans un très petit nombre de cas, à un gonadoblastome (d'origine embryonnaire très proche). En effet, il n'a jamais été observé d'hépatoblastome ou de rhabdomyosarcome chez ces sujets (tableau I).

Peut-être la région du chromosome I I impliquée dans les processus de cancérogénèse touchant ces tissus est différente et correspond à un gène gouvernant un autre stade de la différenciation. Un argument en faveur de cette hypothèse repose sur l'observation, chez certains enfants atteints du syndrome de BeckwithWiedemann, d'une anomalie chromosomique, une duplication cette fois de la région i I pter $\rightarrow$ I I pi 5.4 [23]. Il faut noter toutefois qu'il n'a jamais été observé, jusqu'à présent, de tumeur chez ces enfants (le nombre de cas est faible) et que le mode de transmission de ce syndrome, lorsqu'il est héréditaire, reste mal défini et reflète probablement l'hétérogénéité clinique et génétique de ce syndrome.

\section{Antioncogènes \\ et oncogènes}

Parallèlement aux recherches portant sur les gènes suppresseurs, d'autres groupes ont étudié

\section{* GLOSS AIRE*}

Rhabdomyosarcome: tumeur du muscle strié.

Lymphome de Burkitt : tumeur solide localisée principalement au niveau des maxillaires et de l'abdomen.

Sarcome d'Eming: tumeur osseuse de l'enfant.

Aniridie: absence d'iris.

Omphalocèle: hernie ombilicale.

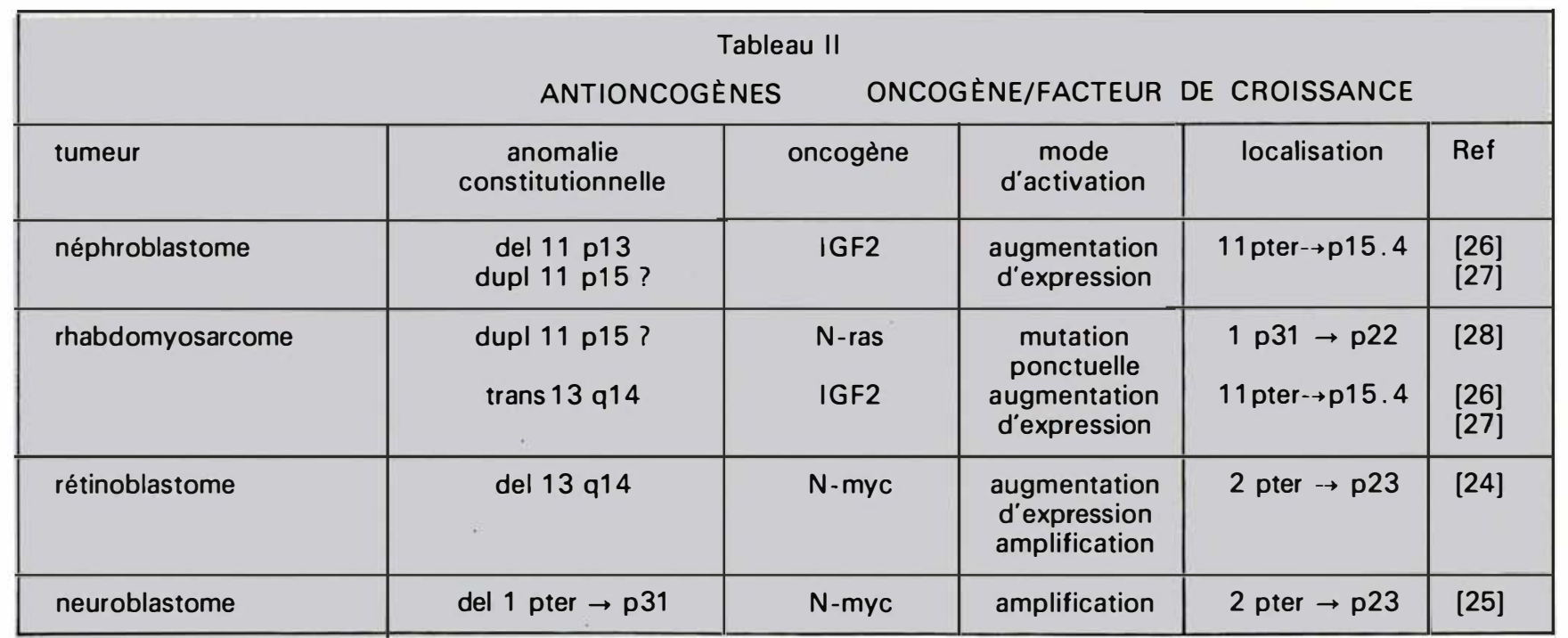

Relations entre oncogènes et antioncogènes. La localisation de gènes suppresseurs impliqués dans ces tumeurs a été connue grâce à l'existence d'anomalies chromosomiques constitutionnelles (ou acquises). La neutralisation par délétion ou mutation des deux allèles pourraient permettre l'expression anormale ou favoriser les mutations d'oncogènes cellulaires ou de facteurs de croissance. La localisation d'IGF2 en 11 pter $\rightarrow 11$ p15. 4, région dupliquée dans le BWS, est un argument en faveur de cette hypothèse. del=délétion; dup/=duplication; trans=translocation. 


\section{RÉFERENCES}

23. Jeanpierre M, Henry I, Turleau C, Ullrich A Grouchy J de, Junien C. Beckwith-Wiedemann syndrome (BWS) and I I p i 5 markers. HGMB, Cytogenet Cell Genet (à paraître).

24. Lee WH, Murphree AL, Benedict WF. Expression of the $\mathrm{N}$-myc gene in primary retinoblastoma. Nature 1984; 309: 458-60.

25. Brodeur GM, Seeger RC, Schwab M, Warmus HE, Bishop JM. Amplification of - N-myc in unitreated human neuroblastomas correlates with advanced discase stage. Science 1984 ; 224: 11 2 I-4

26. Reeve AE, Eccles MR, Wilkins RJ, Bell GI, Millow LJ. Expression of insulin-like growth factor-II transcripts in Wilms'tumour. Nature 1985; 31 7: 258-60.

27. Scott J, Cowell J, Robertson ME, et al. Insulin-like growth factor-II gene expression in Wilms' tumour and embryonic tissues. Nature 1985; 317: 260-2.

28. Chardin $P$, Yeramian $P$, Madaule $P$, Tavitian A. N-ras gene activation in the Rd human rhabdomyosarcoma cell line. Int 7 Cancer 1985; 35: 647-52.

29. Slamon DJ, de Kernion JB, Verma IM, Cline MJ. Expression of cellular oncogenes in human malignancies. Science 1984; 224: 256-62.

30. Dracopoli NC, Houghton AN, Old L. Loss of polymorphic restriction fragments in malignant melanoma: implications for tumor heterogeneity. Proc Natl Acad Sci USA 1985; 82: 1470-4

31. Green AR, Wyke JA. Anti-oncogenes: a subset of regulatory genes involved in carcinogenesis? Lancet $1985 ; 475-7$.

32. Kudson AG Jr. Hereditary Cancer, oncogenes, and antioncogenes. Cancer Res 1985; 45: 1437-43.

33. Erikson J, Williams DL, Finan J, Nowell PC Croce CM. Locus of the $\alpha$ chain of the T-cell receptor is split by chromosome translocation in T-cell leukaemias. Science 1985; 229: 784-6.

34. Lewis WH, Michalopoulos EE, Williams DL, Minden MD, Mak TW. Two T-cell leukaemia patients with çhromosomal translocations have breakpoints within the human $\mathrm{T}$-cell antigen l'expression d'oncogènes dans différents types de tumeurs (tableau II). Dans les tumeurs d'origine neurale, comme le neuroblastome $(\mathrm{Nb})$ et le rétinoblastome (pour lesquels des gènes suppresseurs ont été localisés en $I p$ et $13 q$ respectivement), une augmentation de l'expression du gène $\mathrm{N}$-myc a été notée dans tous les $\mathrm{Rb}$ examinés [24] et dans quelques $\mathrm{Nb}$ [25]. Dans le cas du $\mathrm{Nb}$, cette augmentation de l'expression est parfois associée à une amplification importante du nombre de copies de ce gène qui semble être en corrélation avec le stade de progression tumorale (état métastatique avancé) et conférer un pronostic plus grave.

En revanche, tous les cas de $R b$ examinés correspondaient à des tumeurs primaires relativement petites non métastasées. Il est donc possible que l'augmentation du nombre de copies de $\mathrm{N}$-myc dans le $\mathrm{Nb}$ ne soit pas l'événement primaire, alors que l'augmentation de l'expression de $\mathrm{N}$-myc dans tous les $\mathrm{Rb}$ étudiés attribue à ce gène homologue de l'oncogène myc un rôle déterminant: il semblerait donc logique de penser que le gène $R b^{s}$ participe ou contrôle l'expression de $\mathrm{N}$-myc.

Ainsi, la régulation de l'expression d'un même oncogène pourrait être sous contrôle direct $(\mathrm{Rb})$ ou indirect (Nb) d'au moins deux gènes suppresseurs différents impliqués dans le développement de tumeurs d'origine neurale : le $\mathrm{Nb}^{\mathbf{s}}$ et le $\mathrm{Rb}^{\mathbf{s}}$.

Pour la tumeur de Wilms et le rhabdomyosarcome, dont l'apparition dépend d'un même mécanisme correspondant à la perte d'un gène suppresseur du chromosome II, une augmentation de l'expression du facteur de croissance Insulin-like growth factor II (IGF2) a été mise en évidence récemment [26, 27]. Or, ce facteur de croissance se trouve localisé précisément en I $p$ ter $\rightarrow$ I I p I 5 .4, région dupliquée chez certains sujets atteints de BW. Il est frappant, de plus, de constater que les tissus fœetaux, dans lesquels ce facteur de croissance s'exprime le plus, sont précisément ceux que l'on trouve exagérément développés chez les sujets atteints de BW [20].

L'augmentation d'expression de
N-myc ou d'IGF 2, bien qu'apparemment spécifique, peut n'être qu'un épiphénomene; en effet l'implication d'un oncogène ou d'un facteur de croissance semble rarement réservée à un seul type de tumeur [28]. Il faudrait pouvoir mesurer l'expression de tous les oncogènes et facteurs de croissance connus dans les différents types de tumeur [29]. Il n'est pas certain non plus qu'une combinaison unique de gènes soit concernée pour une tumeur donnée.

De même, bien que la réalisation de l'homozygotie soit un mécanisme beaucoup plus fréquent qu'il n'avait été soupçonné, il n'est pas toujours facile de comprendre les données expérimentales. Dans le cas du mélanome cutané malin, une perte de l'hétérozygotie a été constatée pour 8 chromosomes sur Io, sans qu'il s'agisse du ou des mêmes chromosomes chez les différents sujets étudiés. La perte de l'hétérozygotie est peut-être aussi la conséquence de la progression tumorale [30]. Il n'est pas impossible non plus que différents gènes suppresseurs soient impliqués pour une même tumeur et fonctionnent comme des gènes de contrôle négatif $[31,32]$.

\section{Vers une approche moléculaire}

Récemment, plusieurs groupes $[33,34]$ ont étudié des cas de leucémies à cellules $T$ présentant une translocation t II; I 4(pI3;qII) avec des points de cassure dans le locus des gènes des chaînes $\alpha$ des récepteurs T. Or, la région "receveuse" se trouve sur le chromosome $\mathrm{I} I$, très précisément en I I pr 3. La caractérisation du fragment de jonction apportera peut-être enfin la lumière sur la nature du gène $W^{\mathrm{s}}$, s'il s'agit bien du même gène. Une autre approche devrait également répondre à ces questions: il s'agit de la recherche, grâce à des expériences de transfection, de gènes suppresseurs ou de gènes de résistance à des facteurs transformants.

La découverte de la nature de ces gènes permettra de comprendre une étape supplémentaire liée à l'apparition de tumeurs héréditaires mais aussi vraisemblablement de tumeurs sporadiques 


\section{Diagramme}

\section{du chromosome}

\section{1}

Ce diagramme du chromosome I I indique la région dupliquée dans le syndrome de BeckwithWiedemann (BWS) et la région délétée dans le syndrome WAGR.

$\mathrm{p}=$ bras court du chromosome $\mathrm{q}=$ bras long du chromosome.

Le bras long est subdivisé en deux régions numérotées $\mathrm{I}$ et 2 à partir du centromère, ellesmêmes divisées en bandes et en sous-bandes numérotées de la même façon. Le bras court comprend une seule région (I) divisée en cinq bandes: pi pi2, pi 3, pi 4, pi 5 .

Les points de cassure limitant l'étendue d'un segment impliqué dans une anomalie chromosomique sont notés en commençant par le point de cassure le plus distal (télomérique), suivi du point de cassure proximal (centrométrique). La délétion de la bande pi 3 dans les WAGR s'écrit: del I I pI $4 \rightarrow$ I I pI 3; la duplication du BWS s'écrit: dupl I I pter $\rightarrow$ I I pI 5 . 4 (ter $=$ terminal). Les limites indiquent les bords proximaux des bandes. Dans le cas d'une translocation réciproque entre deux chromosomes, les chromosomes impliqués sont notés par ordre de taille décroissante, puis les points de cassure correspondant respectivement à chacun des chromosomes sont indiqués: la translocation entre le chromosome i i et le chromosome I4 avec points de cassure en I I pI 3 et I 4 q I I, respectivement, s'écrit : t (I I; I4) (pI 3; q I I).

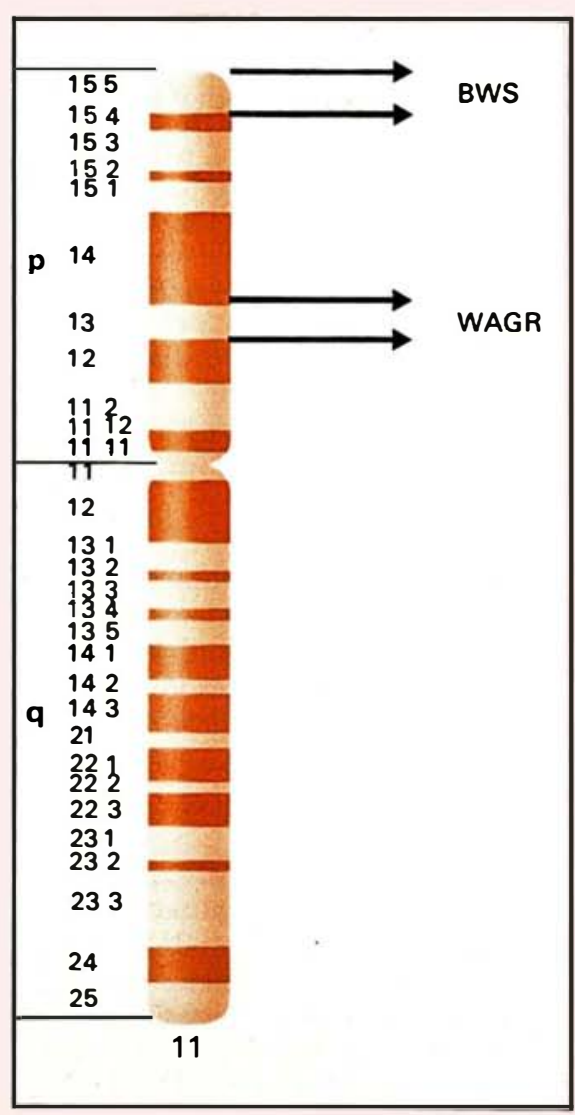

\section{Summary}

Several sets of evidence brought forth by genetic, cytogenetic and molecular findings in hereditary cancers such as retinoblastoma and nephroblastoma support the existence of a new class of genes different from the classical oncogenes, that are also involved in the multistep process of carcinogenesis. In these tumors, which may occur in association with a specific chromosomal deletion, loss or mutation of both copies of genes that lie in these regions could be inferred from molecular studies, thus indicating that recessive mutations can be associated with carcinogenesis.

Furthermore the existence of a pathogenetic pathway common to different types of geneticallyrelated tumors and evidence of uncontrolled expression of cellular oncogenes suggest that these suppressor genes (antioncogenes) may represent a subset of genes important in negative regulatory control of oncogenes and most probably in more general mechanisms of the eucaryotic gene control system at different levels.
TIRÉS A PART

C. Junien: Unité Inserm U 73 et laboratoire de biochimie-génétique, hôpital Ambroise-Paré, Château de Longchamp, Bois de Boulogne, 75016 Paris. 